\title{
UNIVERSAL INFINITE PARTIALLY ORDERED SETS
}

\author{
JOHN B. JOHNSTON
}

Introduction. The purpose of this paper is to construct for each infinite cardinal $\boldsymbol{N}$ an $\boldsymbol{N}$-universal partially ordered set in which every partially ordered set of cardinality $\aleph$ can be embedded. An $\boldsymbol{N}$-universal partially ordered set of cardinality $2^{\boldsymbol{N}}$ is constructed for every infinite cardinal $\boldsymbol{\aleph}$. Furthermore, an $\boldsymbol{\aleph}$-universal partially ordered set of cardinality $\aleph$ is constructed for every cardinal $\aleph$ which is the sum of denumerably many smaller cardinals. The existence for an arbitrary cardinal $\aleph$ of an $\aleph$-universal partially ordered set of cardinality $\boldsymbol{N}$ is left an open question and appears to be a difficult problem.

The ordering relation " $<$ " in a partially ordered set is understood to be transitive, nonreflexive, and nonsymmetric. The Axiom of Choice will be assumed throughout this paper. The General Continuum Hypothesis will be explicitly mentioned when it is used.

1. Totally ordered extensions of partially ordered sets.

Definition: ${ }^{1}$ Let $\alpha \in O n$. If the functions $f: \bar{\omega}_{\alpha} \rightarrow \overline{2}$ are ordered according to first differences, the set $\overline{2}^{\bar{\omega}_{\alpha}}$ becomes a totally ordered set which we denote by $T\left(\overline{2}^{\bar{\omega}_{\alpha}}\right)$. We shall call a function $f: \bar{\omega}_{\alpha} \rightarrow \overline{2}$ an $\alpha$-rational if there exists an ordinal $\eta(f)<\omega_{\alpha}$, necessarily unique, such that $f^{\prime} \eta(f)=1$ while $f^{\prime} \mu=0$ for $\eta(f)<\mu<\omega_{\alpha}$. In the remainder of this paper $\eta$ will be used exclusively to denote this "terminating" ordinal of the $\alpha$-rationals. We denote by $T_{\alpha}$ the totally ordered suborder of $T\left(\overline{2}^{\bar{\omega}}\right)$ consisting of the $\alpha$-rationals.

TheOREM 1. Let $\alpha \in O n . T_{\alpha}$ is a totally ordered set of cardinality $\leqq 2 \aleph_{\alpha}$. Let $\rho<\omega_{\alpha}$ and let $A$ be any subset of $T_{\alpha}$ such that for every $f \in A, \eta(f)<\rho$. Then there exists a (unique) minimal element $h(A, \rho) \in T_{\alpha}$ having the properties that $\eta[h(A, \rho)]=\rho$ and $h(A, \rho)>f$ in $T_{\alpha}$ for every $f \in A$. If $g \in T_{\alpha}$ with $\eta(g)<\rho$ and $g>f$ in $T_{\alpha}$ for every $f \in A$, then $g>h(A, \rho)$ in $T_{\alpha}$. If $\alpha=0$ or if the General Continuum Hypothesis holds, $T_{\alpha}$ has cardinality $\boldsymbol{\aleph}_{\alpha}$.

Received by the editors June 1, 1955.

1 On denotes the class of ordinals. If $\mu$ is an ordinal, $\bar{\mu}$ denotes the class of ordinals less than $\mu$. To show that a function $F$ has a set $R$ for its domain and has its range in a set $S$, we write $F: R \rightarrow S$. For particular sets $R$ and $S$, the set of all functions $F: R \rightarrow S$ is denoted by $S^{R}$. The image of an element $a$ by a function $F$ is denoted by $F^{\prime} a$. 
Proof. (1) $T_{\alpha}=U\left\{B(\eta): \eta<\omega_{\alpha}\right\}$, where $B(\eta)=\left\{f: f \in T_{\alpha}\right.$ and $\eta(f)=\eta\}$ and the $B(\eta)$ are disjoint, so that $\left|T_{\alpha}\right|=\sum\left\{|B(\eta)|: \eta<\omega_{\alpha}\right\}$. Now $|B(\eta)|=2^{|\eta|} \leqq 2^{\aleph_{\alpha}}$, from which we infer that $\left|T_{\alpha}\right| \leqq 2^{\aleph_{\alpha}}$. If $\alpha=0$ or if the General Continuum Hypothesis holds, then $|B(\eta)|$ $\leqq \aleph_{\alpha}$, and we obtain $\left|T_{\alpha}\right|=\boldsymbol{\aleph}_{\alpha}$.

(2) Let $\rho$ and $A$ satisfy the hypothesis of the theorem. We take for $h(A, \rho)$ the unique function $k: \bar{\omega}_{\alpha} \rightarrow \overline{2}$ defined inductively by:

(a) for $\mu<\rho, k^{\prime} \mu=1$ if and only if there exists an $f \in A$ such that $f|\bar{\mu}=k| \bar{\mu}$ and $f^{\prime} \mu=1 ;^{2}$

(b) $k^{\prime} \rho=1$ and $k^{\prime} \mu=0$ for $\rho<\mu<\omega_{\alpha}$.

Clearly, $k \in T_{\alpha}$ and $\eta(k)=\rho$.

(3) Let $f \in A$. Since $\eta(f)<\rho=\eta(k)$, there exists a first ordinal $\sigma \leqq \rho$ for which $f^{\prime} \sigma \neq k^{\prime} \sigma$ while $f|\bar{\sigma}=k| \bar{\sigma}$. If it were true that $f^{\prime} \sigma=1>0=k^{\prime} \sigma$, then necessarily $\sigma<\rho$ and we would have a contradiction of (2). Thus $f^{\prime} \sigma=0<1=k^{\prime} \sigma$ and $f<k$ in $T_{\alpha}$.

(4) Let $g \in T_{\alpha}$ with $\eta(g)=\tau \leqq \rho=\eta(k)$ such that $g<k$ in $T_{\alpha}$. Then there exits a first ordinal $\sigma \leqq \rho$ such that $g^{\prime} \sigma=0<1=k^{\prime} \sigma$ while $g|\bar{\sigma}=k| \bar{\sigma}$. Note that not both $\sigma=\rho$ and $\tau=\rho$.

(a) Suppose that $\sigma<\rho$. Then by (2), $k^{\prime} \sigma=1$ implies that there exists an $f \in A$ such that $f|\bar{\sigma}=k| \bar{\sigma}=g \mid \bar{\sigma}$ while $f^{\prime} \sigma=k^{\prime} \sigma=1>0=g^{\prime} \sigma$. For this $f \in A$ we have $f>g$ in $T_{\alpha}$.

(b) Suppose that $\tau<\sigma=\rho$. Then by (2), $k^{\prime} \tau=g^{\prime} \tau=1$ implies that there exists an $f \in A$ such that $f|\bar{\tau}=k| \bar{\tau}=g \mid \bar{\tau}$ while $f^{\prime} \tau=k^{\prime} \tau=g^{\prime} \tau=1$. Since $\eta(g)=\tau$, we have $g \leqq f$ in $T_{\alpha}$ for this particular $f$.

The minimality of $h(A, \rho)=k$ follows from (a) with $\tau=\rho$. For $g \in T_{\alpha}$ with $\eta(g)<\rho$ and $g>f$ in $T_{\alpha}$ for every $f \in A$, the fact that $g>k$ in $T_{\alpha}$ follows from (b) and (a) with $\tau<\rho$, since $\eta(g)<\rho$ implies that $g \neq k$. This completes the proof of Theorem 1 .

If $P$ is a partially ordered set and $T$ a totally ordered set, a biunique function $F: P \rightarrow T$ is said to generate a totally ordered extension of $P$ if for any two elements $a$ and $b$ of $P, a<b$ in $P$ implies that $F^{\prime} a<F^{\prime} b$ in $T$. The totally ordered extension generated is the set of elements of $P$ together with the order relations transferred from $T$ back to $P$ by the inverse of $F$. If $P$ is itself a totally ordered set, then $P$ is similar to the totally ordered set $\left\{F^{\prime} a: a \in P\right\}$ and $F$ is called an embedding of $P$ in $T$.

THEOREM 2. Let $\alpha \in O n$ and let $P$ be any partially ordered set of cardinality $\aleph_{\alpha}$ with an imposed well-ordering, $P=\left\{p(\mu): \mu<\omega_{\alpha}\right\}$. For $\nu \leqq \omega_{\alpha}$, define $P_{\nu}=\{p(\mu): \mu<\nu\}$. Let $0 \leqq \rho<\sigma \leqq \omega_{\alpha}$ and suppose that $G$ :

2 If $F$ is a function and $R$ is a subset of its domain, $F \mid R$ denotes the function $F$ restricted to the domain $R$. 
$P_{\rho} \rightarrow T_{\alpha}$ is any function which generates a totally ordered extension of $P_{\rho}$ in such a manner that for each $p(\mu) \in P_{\rho}, \eta\left[G^{\prime} p(\mu)\right]=\mu$. By induction there exists a unique function $F: P_{\sigma} \rightarrow T_{\alpha}$ such that $F \mid P_{\rho}=G$ while $F^{\prime} p(\nu)=h\left(A_{\nu}, \nu\right)$ for $\rho \leqq \nu<\sigma$, where $A_{\nu}=\left\{F^{\prime} p(\mu): \mu<\nu\right.$ and $p(\mu)<p(\nu)$ in $P\}$. We conclude that this function $F$ generates a totally ordered extension of $P_{\sigma}$ in such a manner that for each $p(\mu) \in P_{\sigma}, \eta\left[F^{\prime} p(\mu)\right]=\mu$. We denote the function $F \mid\left(P_{\sigma}-P_{\rho}\right)$ by $F(G, \sigma)$.

Proof. (1) Let $\mu, \nu<\rho$ with $p(\mu)<p(\nu)$ in $P$. Then $F^{\prime} p(\mu)=G^{\prime} p(\mu)$ $<G^{\prime} p(\nu)=F^{\prime} p(\nu)$ in $T_{\alpha}$ by the assumed property of $G$.

(2) Let $\mu<\nu<\sigma$ with $\nu \geqq \rho$ and $p(\mu)<p(\nu)$ in $P$. Then $F^{\prime} p(\mu) \in A$, so that $F^{\prime} p(\nu)=h\left(A_{\nu}, \nu\right)>F^{\prime} p(\mu)$ in $T_{\alpha}$ by Theorem 1 .

(3) Let $\mu<\nu<\sigma$ with $\nu \geqq \rho$ and $p(\mu)>p(\nu)$ in $P$. Suppose it were true that $F^{\prime} p(\mu)<F^{\prime} p(\nu)$ in $T_{\alpha}$. Let $\tau$ be the first such ordinal $\nu$. Then $p(\mu)>p(\tau)$ in $P$ implies that $p(\mu)>p(\pi)$ in $P$ for every $\pi<\tau$ for which $p(\tau)>p(\pi)$ in $P$. If $\pi<\mu$ then $F^{\prime} p(\pi)<F^{\prime} p(\mu)$ in $T_{\alpha}$ by (1) and (2). If $\mu<\pi<\rho$ then by (1), $F^{\prime} p(\pi)<F^{\prime} p(\mu)$ in $T_{\alpha}$. If $\mu<\pi<\tau$ and $\pi \geqq \rho$ then by the minimality of $\tau, F^{\prime} p(\pi)<F^{\prime} p(\mu)$ in $T_{\alpha}$. Thus for every $f \in A_{\tau}$ we have $F^{\prime} p(\mu)>f$ in $T_{\alpha}$ so that by Theorem $1, F^{\prime} p(\mu)$ $>h\left(A_{\tau}, \tau\right)=F^{\prime} p(\tau)$ in $T_{\alpha}$, contrary to our supposition. So, for $\mu<\nu<\sigma$ with $\nu \geqq \rho$ and $p(\mu)>p(\nu)$ in $P$, we have $F^{\prime} p(\mu)>F^{\prime} p(\nu)$ in $T_{\alpha}$, which completes the proof of Theorem 2 .

For each $\alpha \in O n$ we say that a totally ordered set $T$ is $\boldsymbol{\aleph}_{\alpha}$-universal if every totally ordered set of cardinality $\boldsymbol{\aleph}_{\alpha}$ has an embedding in $T$.

If in Theorem 2 we take $P$ to be any totally ordered set of cardinality $\boldsymbol{\aleph}_{\alpha}$ and set $0=\rho$ and $\sigma=\omega_{\alpha}$, we obtain immediately the theorem of N. Cuesta Dutari [2, p. 243, Theorem 15]: For each $\alpha \in O n, T_{\alpha}$ is an $\aleph_{\alpha}$-universal totally ordered set of cardinality $\leqq 2 \aleph_{\alpha}$; if $\alpha=0$ or if the General Continuum Hypothesis holds then $T_{\alpha}$ has cardinality $\boldsymbol{\aleph}_{\alpha}$. This theorem has also been obtained for nonlimit cardinals $\boldsymbol{\aleph}_{\alpha}$ by W. Sierpinski [6, p. 62, Theorem 3], using a theorem due to F. Hausdorff $[3$, pp. 181-182]; the method of proof could be extended to regular cardinals but not to singular cardinals.

Suppose that $P$ is a partially ordered set of cardinality $\boldsymbol{\aleph}_{\alpha}$ having distinct noncomparable elements $a$ and $b$. If we impose on $P$ a wellordering, $P=\left\{p(\mu): \mu<\omega_{\alpha}\right\}$, in which $p(0)=b$ and $p(1)=a$, and if we set $0=\rho$ and $\sigma=\omega_{\alpha}$, then Theorem 2 yields immediately the theorem of E. Szpilrajn [7]: If $P$ is a partially ordered set having distinct noncomparable elements $a$ and $b$, then there exists a totally ordered extension of $P$ in which $a<b$.

2. Universal infinite partially ordered sets. If $P$ and $Q$ are partially ordered sets, a bi-unique function $F: P \rightarrow Q$ is called an embedding of 
$P$ in $Q$ if for every two elements $a$ and $b$ of $P, a<b$ in $P$ if and only if $F^{\prime} a<F^{\prime} b$ in $Q$.

Let $P$ be a partially ordered set. A set $K$ of totally ordered extensions of $P$ is said to realize $P$ if for any two elements $a$ and $b$ of $P$, $a<b$ in $P$ if and only if $a<b$ in every member of $K$. By the theorem of E. Szpilrajn [7], the set of all totally ordered extensions of $P$ realizes $P$. Thus, among such realizing sets $K$ there will be one of minimal cardinality $(\geqq 1)$. B. Dushnik and E. W. Miller [1] call this minimal cardinal the dimension of $P$ and prove the following statements:

$\left(^{*}\right)$ Every infinite partially ordered set has a dimension less than or equal to its cardinality.

(**) If a partially ordered set $P$ has an embedding in a partially ordered set $Q$, then dimension $P \leqq$ dimension $Q$.

(***) For $\alpha \in O n$ and any cardinal $\mathfrak{m}$ satisfying $0<\mathfrak{m} \leqq \boldsymbol{\aleph}_{\alpha}$, there exists a partially ordered set of cardinality $\boldsymbol{\aleph}_{\alpha}$ and dimension $\mathfrak{m}$.

For each $\alpha \in O n$, we say that a partially ordered set $P$ is $\boldsymbol{\aleph}_{\alpha}$-universal if every partially ordered set of cardinality $\boldsymbol{\aleph}_{\alpha}$ has an embedding in $P$; we say that $P$ is $\boldsymbol{\aleph}_{\alpha}$-sub-universal if every partially ordered set of cardinality $\boldsymbol{\aleph}_{\alpha}$ and dimension $\left\langle\boldsymbol{\aleph}_{\alpha}\right.$ has an embedding in $P$.

Definition. Let $\alpha \in O n$. The set $T_{\alpha^{\alpha}}^{\bar{\omega}}$ clearly becomes a partially ordered set, which we denote by $P\left(T_{\alpha}^{\bar{\omega}_{\alpha}}\right)$, if we order the functions $F: \bar{\omega}_{\alpha} \rightarrow T_{\alpha}$ as follows: for $F_{1}, F_{2} \in T_{\alpha}^{\bar{\omega}}, F_{1}<F_{2}$ in $P\left(T_{\alpha}^{\bar{\omega}}\right)$ if and only if for every $\mu<\omega_{\alpha}, F_{1}^{\prime} \mu<F_{2}^{\prime} \mu$ in $T_{\alpha}$. A function $F: \bar{\omega}_{\alpha} \rightarrow T_{\alpha}$ is said to be of constant degree if there exists an ordinal $\delta<\omega_{\alpha}$ such that for every $\mu<\omega_{\alpha}, \eta\left[F^{\prime} \mu\right]=\delta$; this ordinal $\delta$ is denoted by $\Delta(F)$. A function $F: \bar{\omega}_{\alpha} \rightarrow T_{\alpha}$ is said to be periodic if there exists an ordinal $\pi$ with $0<\pi<\omega_{\alpha}$ such that for every $\mu<\omega_{\alpha}, F^{\prime} \mu=F^{\prime} \Phi(\pi, \mu) ;^{3}$ the minimal such $\pi$ is denoted by $\Pi(F) . P\left(\boldsymbol{\aleph}_{\alpha}\right)$ will denote the partially ordered suborder of $P\left(T_{\alpha}^{\bar{\omega}}\right)$ consisting of the functions of constant degree. $P_{\alpha}$ will denote the partially ordered suborder of $P\left(\boldsymbol{N}_{\alpha}\right)$ consisting of the periodic functions of constant degree.

Theorem 3. Let $\alpha \in O n$. Then $P\left(\boldsymbol{\aleph}_{\alpha}\right)$ is an $\boldsymbol{\aleph}_{\alpha}$-universal partially ordered set of cardinality $2 \boldsymbol{\aleph}_{\alpha}$ and dimension $\boldsymbol{\aleph}_{\alpha} . P_{\alpha}$ is an $\boldsymbol{\aleph}_{\alpha}$-subuniversal partially ordered set of cardinality $\leqq 2 \aleph_{\alpha}$ for which $\lim \left\{\mathfrak{m}: \mathfrak{m}<\boldsymbol{\aleph}_{\alpha}\right\} \leqq$ dimension $P_{\alpha} \leqq \boldsymbol{\aleph}_{\alpha} ;$ if $\alpha=0$ or if the General Continuum Hypothesis holds, then $P_{\alpha}$ has cardinality $\boldsymbol{\aleph}_{\alpha}$.

\footnotetext{
3 If $\mu$ and $\nu$ are ordinals with $\mu>0$, then there exist unique ordinals $\Theta(\mu, \nu)$ and $\Phi(\mu, \nu)$ with $\Theta(\mu, \nu) \leqq \nu$ and $\Phi(\mu, \nu)<\mu$ such that $\nu$ has the representation: $\nu=\mu \times \Theta(\mu, \nu)$ $+\Phi(\mu, \nu)$.
} 
Proof. (1) $P\left(\boldsymbol{\aleph}_{\alpha}\right)=\bigcup\left\{B(\delta): \delta<\omega_{\alpha}\right\}$, where $B(\delta)=\left\{F: F \in P\left(\boldsymbol{\aleph}_{\alpha}\right)\right.$ and $\Delta(F)=\delta\}$ and the $B(\delta)$ are disjoint, so that $\left|P\left(\boldsymbol{\aleph}_{\alpha}\right)\right|$

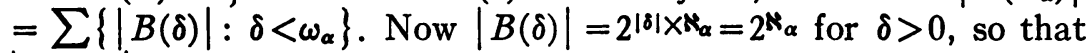
$\left|P\left(\boldsymbol{\aleph}_{\alpha}\right)\right|=2 \boldsymbol{\aleph}_{\alpha}$.

(2) $P_{\alpha}=\bigcup\left\{B(\delta, \pi): \delta<\omega_{\alpha}, \pi<\omega_{\alpha}\right\}$, where $B(\delta, \pi)=\left\{F: F \in P_{\alpha}\right.$, $\Delta(F)=\delta$, and $\left.\prod(F)=\pi\right\}$ and the $B(\delta, \pi)$ are disjoint, so that $\left|P_{\alpha}\right|=\sum\left\{|B(\delta, \pi)|: \delta<\omega_{\alpha}, \pi<\omega_{\alpha}\right\}$. Now $|B(\delta, \pi)|=2^{|\delta| \times|\pi|} \leqq 2^{\boldsymbol{N}_{\alpha}}$, so that $\left|P_{\alpha}\right| \leqq 2^{\aleph_{\alpha}}$. If $\alpha=0$ or if the General Continuum Hypothesis holds, then $|\bar{B}(\delta, \pi)| \leqq \aleph_{\alpha}$ and we obtain $\left|P_{\alpha}\right|=\aleph_{\alpha}$.

(3) Let $P$ be any partially ordered set of cardinality $\boldsymbol{\aleph}_{\alpha}$ with an imposed well-ordering, $P=\left\{p(\mu): \mu<\omega_{\alpha}\right\}$. Let $K$ be a realizing set of totally ordered extensions of $P$ with $|K|=\operatorname{dimension} P$ so that we may write $K=\{T(\nu): \nu<\zeta\}$, where $\zeta$ is the initial ordinal of dimension $P$ and $\zeta \leqq \omega_{\alpha}$ by $\left({ }^{*}\right)$. For $\nu<\zeta$, define $G(\nu): T(\nu) \rightarrow T_{\alpha}$ to be the function $F$ of Theorem 2 for the totally ordered set $T(\nu)$ $=\left\{p(\mu): \mu<\omega_{\alpha}\right\}$, taking $0=\rho$ and $\sigma=\omega_{\alpha}$. If $\zeta<\omega_{\alpha}$, define $G(\nu)$ $=G[\Phi(\zeta, \nu)]$ for $\zeta \leqq \nu<\omega_{\alpha}$. For each $\mu<\omega_{\alpha}$ we define $F(\mu): \bar{\omega}_{\alpha} \rightarrow T_{\alpha}$ by setting $F(\mu)^{\prime} \nu=G(\nu)^{\prime} p(\mu)$ for each $\nu<\omega_{\alpha}$. Finally we define $F$ : $P \rightarrow P\left(T_{\alpha}^{\overline{\omega_{\alpha}}}\right)$ by setting $F^{\prime} p(\mu)=F(\mu)$ for each $\mu<\omega_{\alpha}$. By the assumed property of $K$ and the above constructions, $F$ is an embedding of $P$ in $P\left(\boldsymbol{\aleph}_{\alpha}\right)$; furthermore, if dimension $P<\aleph_{\alpha}$, then $\zeta<\omega_{\alpha}$ and $F$ is an embedding of $P$ in $P_{\alpha}$.

(4) By $\left({ }^{* *}\right),\left({ }^{* *}\right)$, and step (3) above, it remains only to exhibit a set of $\boldsymbol{\aleph}_{\alpha}$ totally ordered extensions of $P\left(\boldsymbol{\aleph}_{\alpha}\right)$ which realizes $P\left(\boldsymbol{\aleph}_{\alpha}\right)$. We define totally ordered extensions $L(\mu), \mu<\omega_{\alpha}$, of $P\left(\boldsymbol{\aleph}_{\alpha}\right)$ as follows. For distinct elements $F_{1}$ and $F_{2}$ of $P\left(\boldsymbol{\aleph}_{\alpha}\right)$ we set $F_{1}<F_{2}$ in $L(\mu)$ if and only if:

(a) $F_{1}^{\prime} \mu<F_{2}^{\prime} \mu$ in $T_{\alpha}$ or,

(b) $F_{1}^{\prime} \mu=F_{2}^{\prime} \mu$ and $F_{1}^{\prime \prime} \nu>F_{2}^{\prime} \nu$ in $T_{\alpha}$ for the first ordinal $\nu<\omega_{\alpha}$ for which $F_{1}^{\prime} \nu \neq F_{2}^{\prime} \nu$.

Each $L(\mu)$ is easily seen to be a totally ordered extension of $P\left(\boldsymbol{\aleph}_{\alpha}\right)$. To show that $\left\{L(\mu): \mu<\omega_{\alpha}\right\}$ realizes $P\left(\boldsymbol{\aleph}_{\alpha}\right)$, we need only consider noncomparable elements of $P\left(\boldsymbol{\aleph}_{\alpha}\right)$. Let $F_{1}$ and $F_{2}$ be distinct noncomparable elements of $P\left(\boldsymbol{\aleph}_{\alpha}\right)$. Since $F_{1}$ and $F_{2}$ are distinct, there exists a first ordinal $\rho<\omega_{\alpha}$ such that $F_{1}^{\prime} p \neq F_{2}^{\prime} p$, and we may assume that $F_{1}^{\prime} p<F_{2}^{\prime} p$ in $T_{\alpha}$ so that $F_{1}<F_{2}$ in $L(\rho)$. Since $F_{1}$ and $F_{2}$ are noncomparable in $P\left(\boldsymbol{N}_{\alpha}\right)$, there exists an ordinal $\sigma<\omega_{\alpha}$ with $\sigma \neq \rho$ for which $F_{1}^{\prime} \sigma \geqq F_{2}^{\prime} \sigma$ in $T_{\alpha}$. If $F_{1}^{\prime} \sigma>F_{2}^{\prime} \sigma$ in $T_{\alpha}$, then $F_{1}>F_{2}$ in $L(\sigma)$. If $F_{1}^{\prime} \sigma=F_{2}^{\prime} \sigma$, then, since $\rho$ is the first ordinal $\nu<\omega_{\alpha}$ for which $F_{1}^{\prime} \nu \neq F_{2}^{\prime} \nu$ and $F_{1}^{\prime} p<F_{2}^{\prime} \rho$ in $T_{\alpha}$, we again have $F_{1}>F_{2}$ in $L(\sigma)$. Thus $\left\{L(\mu): \mu<\omega_{\alpha}\right\}$ realizes $P\left(\boldsymbol{\aleph}_{\alpha}\right)$, completing the proof of Theorem 3 .

Since a totally ordered set is a partially ordered set of dimension 1 , 
Theorem 3 is a generalization of the theorem of N. Cuesta Dutari [2, p. 243, Theorem 15]. We also note that step (4) of the proof of Theorem 3 is a direct generalization of the corresponding step given by $\mathrm{H}$. Komm [4, pp. 510-511] in his proof of essentially the same theorem for the case $\boldsymbol{\aleph}_{\alpha}=\boldsymbol{\aleph}_{0}$.

Theorem 4. If $\lambda=0$, or if the General Continuum Hypothesis holds and $\lambda$ is the limit of a denumerable increasing sequence of smaller ordinals, then $P_{\lambda}$ is an $\aleph_{\lambda}$-universal partially ordered set of cardinality and dimension $\boldsymbol{N}_{\lambda}$.

Proof. (1) $P_{\lambda}$ has cardinality and dimension $\boldsymbol{N}_{\lambda}$ by Theorem 3 .

(2) Let $P$ be any partially ordered set of cardinality $\boldsymbol{N}_{\lambda}$ with an imposed well-ordering, $P=\left\{p(\mu): \mu<\omega_{\alpha}\right\}$. We define subsets $P(n)$ and $P_{n}$ of $P$ for each $n<\omega_{0}$ as follows. If $\lambda=0, P(n)=\{p(n)\}$. If $\lambda>0$, let $\lambda=\lim \left\{\lambda(n): n<\omega_{0}\right\}$, where $\lambda(n)<\lambda(n+1)$ for each $n<\omega_{0}$, so that $\omega_{\lambda(n)}<\omega_{\lambda(n+1)}$ and $\omega_{\lambda(n)+1}<\omega_{\lambda(n+1)+1}$ for each $n<\omega_{0}$ and $\omega_{\lambda}=\lim \left\{\omega_{\lambda(n)}: \quad n<\omega_{0}\right\}=\lim \left\{\omega_{\lambda(n)+1}: \quad n<\omega_{0}\right\}$. We set $P(0)$ $=\left\{p(\mu): \mu<\omega_{\lambda(0)}\right\} ;$ for $0<n<\omega_{0}$ we set $P(n)=\left\{p(\mu): \omega_{\lambda(n-1)} \leqq \mu\right.$ $\left.<\omega_{\lambda(n)}\right\}$. For every value of $\lambda$ we set $P_{n}=U\{P(r): r \leqq n\}$; we note that $P=\bigcup\left\{P(n): n<\omega_{0}\right\}$.

(3) For each $n<\omega_{0}$, define $W(n)=\left\{f: f \in T_{\lambda}^{P(n)}\right.$ and $\eta\left[f^{\prime} p(\mu)\right]=\mu$ for each $p(\mu) \in P(n)\}$. If $\lambda=0,|W(n)|=2^{n}$ and we may write $W(n)=\left\{f(n, \mu): \mu<2^{n}\right\}$ with the convention that $f(n, \mu)^{\prime} p(n)$ $<f(n, \mu+1)^{\prime} p(n)$ in $T_{0}$ for $\mu+1<2^{n}$. If $\lambda>0,|W(n)|=\prod\left\{2^{|\mu|}\right.$ : $p(\mu) \in P(n)\}=2^{\langle n\rangle}$, where $\langle n\rangle=\sum\{|\mu|: p(\mu) \in P(n)\}=\boldsymbol{\aleph}_{\lambda(n)}$ so that $|W(n)|=\boldsymbol{\aleph}_{\lambda(n)+1}$; thus we may well-order $W(n)$ and write $W(n)=\left\{f(n, \mu): \mu<\omega_{\lambda(n)+1}\right\}$.

(4) We define functions $g(n, \zeta): P(n) \rightarrow T_{\lambda}$ for each $n<\omega_{0}$ and for each $\zeta<\omega_{\lambda}$ as follows:

(a) Suppose that $\lambda=0$. We set $g(0, \zeta)=f(0,0)$ for every $\zeta<\omega_{0}$. For $0<n<\omega_{0}$ and $\zeta<\omega_{0}$, define $\zeta(n)=\Phi\left(2^{n(n+1) / 2}, \zeta\right)$ and $\zeta(n, n)$ $=\Theta\left[2^{n(n-1) / 2}, \zeta(n)\right]$, so that $\zeta(n)<2^{n(n+1) / 2}$ and $\zeta(n)=2^{n(n-1) / 2}$ $\times \zeta(n, n)+r$ with $r<2^{n(n-1) / 2}$ and $\zeta(n, n)<2^{n}$; we set $g(n, \zeta)$ $=f[n, \zeta(n, n)]$.

(b) Suppose that $\lambda>0$. We set $g(0, \zeta)=f[0, \zeta(0)]$ for every $\zeta<\omega_{\lambda}$, where $\zeta(0)=\Phi\left(\omega_{\lambda(0)+1}, \zeta\right)$. For $0<n<\omega_{0}$ and $\zeta<\omega_{\lambda}$, define $\zeta(n)=\Phi\left(\omega_{\lambda(n)+1}, \zeta\right)$ and $\zeta(n, n)=\Theta\left[\omega_{\lambda(n-1)+1}, \zeta(n)\right]$, so that $\zeta(n)$ $<\omega_{\lambda(n)+1}$ and $\zeta(n)=\omega_{\lambda(n-1)+1} \times \zeta(n, n)+\mu$ with $\mu<\omega_{\lambda(n-1)+1}$ and $\zeta(n, n)<\omega_{\lambda(n)+1}$; we set $g(n, \zeta)=f[n, \zeta(n, n)]$.

(5) We show by induction on $n$ that if $n<\omega_{0}$ and $G: P_{n} \rightarrow T_{\lambda}$ is any function such that for each $p(\mu) \in P_{n}, \eta\left[G^{\prime} p(\mu)\right]=\mu$, then there exists an ordinal $\gamma(G)<\omega_{\lambda}$ such that $G=\bigcup\{g[r, \gamma(G)]: r \leqq n\}$. If $\lambda=0$, $\gamma(G)$ can be taken $<2^{n(n+1) / 2}$; if $\lambda>0, \gamma(G)$ can be taken $<\omega_{\lambda(n)+1}$. 
This statement is clearly true for $n=0$; let $0<n<\omega_{0}$ and assume that the statement holds for $n-1$. Define $H=G \mid P_{n-1}$. Then for each $p(\mu) \in P_{n-1}, \eta\left[H^{\prime} p(\mu)\right]=\mu$ so that by our inductive assumption, there exists a $\gamma(H)$; if $\lambda=0, \gamma(H)<2^{n(n-1) / 2}$; if $\lambda>0, \gamma(H)<\omega_{\lambda(n-1)+1}$; we have $H=\bigcup\{g[r, \gamma(H)]: r<n\}$. Now $G \mid P(n)$ is a function $G \mid P(n)$ : $P(n) \rightarrow T_{\lambda}$ such that for each $p(\mu) \in P(n), \eta\left[G \mid P(n)^{\prime} p(\mu)\right]=\mu$ so that there exists a $\zeta<\omega_{\lambda}$ for which $G \mid P(n)=f(n, \zeta)$; if $\lambda=0, \zeta<2^{n}$; if $\lambda>0, \zeta<\omega_{\lambda(n)+1}$. We define an ordinal $\gamma$ as follows: if $\gamma=0, \gamma=2^{n(n-1) / 2}$ $\times \zeta+\gamma(H)$; if $\lambda>0, \gamma=\omega_{\lambda(n-1)+1} \times \zeta+\gamma(H)$. Then for $\lambda=0, \lambda<2^{n(n+1) / 2}$, while for $\lambda>0, \gamma<\omega_{\lambda(n)+1}$. Clearly, for $r<n, g(r, \gamma)=g[r, \gamma(H)]$, while $g(n, \gamma)=f(n, \zeta)$. Thus $G=\bigcup\{g(r, \gamma): r \leqq n\}$ and we may take $\gamma(G)=\gamma$.

(6) Now, depending on the order relations in $P$, we define functions $k(n, \zeta): P(n) \rightarrow T_{\lambda}$ for every $n<\omega_{0}$ and every $\zeta<\omega_{\lambda}$ by induction on $n$. Let $n<\omega_{0}$ and $\zeta<\omega_{\lambda}$ and suppose that we have defined functions $k(r, \zeta): P(r) \rightarrow T_{\lambda}$ for every $r<n$ in such a manner that for each $r<n$ and each $p(\mu) \in P(r), \eta\left[k(r, \zeta)^{\prime} p(\mu)\right]=\mu$, and that $U\{k(r, \zeta): r<n\}$ generates a totally ordered extension of $P_{n-1}$ (where by convention we take $P_{-1}$ to be the void set). If $U\{k(r, \zeta): r<n\} \bigcup_{g}(n, \zeta)$ generates a totally ordered extension of $P_{n}$ we set $k(n, \zeta)=g(n, \zeta)$; otherwise we set $k(n, \zeta)=F[\bigcup\{k(r, \zeta): r<n\}, \Omega(n)]$, the function of Theorem 2 for the present partially ordered set $P$, where $\Omega(n)=n+1$ if $\lambda=0$ and $\Omega(n)=\omega_{\lambda(n)}$ if $\lambda>0$. By induction, if $U\{g(r, \zeta): r \leqq n\}$ generates a totally ordered extension of $P_{n}$, then $k(r, \zeta)=g(r, \zeta)$ for every $r \leqq n$. Also by induction, for each $\zeta<\omega_{\lambda}, U\left\{k(n, \zeta): n<\omega_{0}\right\}$ generates a totally ordered extension of $P$.

(7) We define functions $F(\mu): \bar{\omega}_{\lambda} \rightarrow T_{\lambda}$ for each $\mu<\omega_{\lambda}$ by setting $F(\mu)^{\prime} \zeta=k(n, \zeta)^{\prime} p(\mu)$ for each $\zeta<\omega_{\lambda}$, where $n$ is the integer such that $p(\mu) \in P(n)$. By (4) and (6), each $F(\mu) \in P_{\lambda}$ and $\Delta[F(\mu)]=\mu$. We define the desired function $F: P \rightarrow P_{\lambda}$ by setting $F^{\prime} p(\mu)=F(\mu)$ for each $\mu<\omega_{\lambda}$. By the last remark in (6), we need only check that $F$ preserves the noncomparabilities in $P$.

(8) Let $n<\omega_{0}$ and let $G: P_{n} \rightarrow T_{\lambda}$ be any function which generates a totally ordered extension of $P_{n}$ in such a manner that for each $p(\mu) \in P_{n}, \eta\left[G^{\prime} p(\mu)\right]=\mu$. By $(5)$, there exists an ordinal $\gamma(G)<\omega_{\lambda}$ such that $G=\bigcup\{g[r, \gamma(G)]: r \leqq n\}$. But by the next to last sentence of (6), $k[r, \gamma(G)]=g[r, \gamma(G)]$ for $r \leqq n$, so that $G=\bigcup\{k[r, \gamma(G)]: r \leqq n\}$. But now any noncomparability in $P$ can be realized by the extensions $U\left\{k\left[n, \gamma\left(G_{1}\right)\right]: n<\omega_{0}\right\}$ and $U\left\{k\left[n, \gamma\left(G_{2}\right)\right]: n<\omega_{0}\right\}$ of two such functions $G_{1}$ and $G_{2}$ by Theorem 2 and the theorem of E. Szpilrajn [7]. Thus $F$ is the desired embedding for Theorem 4 .

Theorem 4 with $\lambda=0$ has previously been obtained by A. Mostowski [5]. We could, of course, perform a construction analogous to that 
in Theorem 4 for any limit cardinal, but we could not make the statement in step (5) of the proof once $n$ had reached $\omega_{0}$. Step (5) was crucial in proving step (8), which assures us that the function $F$ is actually an embedding. For nonlimit cardinals, Theorem 3 is the best result the author has been able to obtain.

\section{REFERENCES}

1. B. Dushnik and E. W. Miller, Partially ordered sets, Amer. J. Math. vol. 63 (1941) pp. 600-610.

2. N. Cuesta Dutari, Teoria decimal de los tipos de orden, Revista Matematica Hispano-Americana vol. 3 (1943) pp. 186-205.

3. F. Hausdorff, Grundzilge der Mengenlehre, Leipzig, 1914.

4. H. Komm, On the dimension of partially ordered sets, Amer. J. Math. vol. 70 (1948) pp. 507-520.

5. A. Mostowski, Über gewisse universelle Relationen, Annales de la Société Polonaise de Mathématique vol. 17 (1938) pp. 117-118.

6. W. Sierpinski, Sur une propriete des ensembles ordonnes, Fund. Math. vol. 36 (1949) pp. 56-67.

7. E. Szpilrajn, Sur l'extension de l'ordre partiel, Fund. Math. vol. 16 (1930) pp. 386-389.

California Institute of Technology 